\title{
Estudo epidemiológico das taxas de internação hospitalar entre a mastectomia oncológica e a plástica mamária reconstrutiva pós-mastectomia no período de junho de 2015 a junho de 2020, no Estado do Rio de Janeiro
}

Epidemiological study of hospital admission rates between oncological mastectomy and post-mastectomy reconstructive breast plastic surgery from June 2015 to June 2020, in the state from Rio de Janeiro

Tayná Vieira Pires ${ }^{1 *}$, Alan Ferreira Silva ${ }^{2}$, Sarah Lages Coelho $^{3}$, Suzane Aguiar de Souza ${ }^{4}$, Sebastião Jorge da Cunha Golçalves $^{5}$

Como citar esse artigo. Pires, T.V; Silva, A.F; Coelho, S.L; de Souza, S.A; Gonçalves, S.J.C. Estudo epidemiológico das taxas de internação hospitala entre a mastectomia em oncologia e a plástica mamári reconstrutiva pós mastectomia no período de junho $\mathrm{d}$ 2015 a junho de 2020, no Estado do Rio de Janeiro. Revista de Saúde. 2021 Abr./Jul.; 12 (2): 45-48.

\section{Resumo}

O câncer de mama é um importante problema de saúde pública e a neoplasia maligna mais incidente em mulheres no Brasil. Uma vez feito o diagnóstico, o principal tratamento efetivo é através da cirurgia, podendo interferir no estado emocional da paciente já que a mama é um importante símbolo ligado a feminilidade. O objetivo da pesquisa é estudar o número de internações hospitalares entre a mastectomia oncológica e a plástica mamária reconstrutiva pós-mastectomia, a partir de uma pesquisa do tipo epidemiológica descritiva. Foram analisados dados do DATASUS a respeito do número de internações aprovadas e o valor total gasto com tratamento em pacientes submetidas a mastectomia simples em oncologia e mastectomia radical com linfadenectomia axilar em oncologia no período de junho de 2015 a junho de 2020 e pacientes submetidas a plástica mamária reconstrutiva pós-mastectomia com implante de prótese no período de junho de 2015 a junho de 2020. Os dados obtidos através do DATASUS revelam um total de 6.693 internações para realização do tratamento cirúrgico do câncer de mama e 1.185 internações para a realização de plástica mamária reconstrutiva pósmastectomia com implante de prótese, mostrando que existe uma baixa procura para a cirurgia mamária reconstrutiva pelo Sistema Único de Saúde.A mastectomia se trata de um processo invasivo, que acarreta na mutilação da mama, comprometendo a autoimagem corporal e a autoestima da paciente. Nesse contexto, a cirurgia reconstrutiva reduz os estigmas e sequelas deixadas pela mastectomia.

Palavras-chave: Mastectomia;Mamoplastia; Neoplasias mamárias; Qualidade de vida.

\begin{abstract}
Breast cancer is an important public health problem and the most common malignancy in women in Brazil. Once the diagnosis is made, the main effective treatment is through surgery, which can interfere with the patient's emotional state since the breast is an important symbol linked to femininity. The objective of the research is to study the number of hospital admissions between oncologicalmastectomyand reconstructive breast plastic surgery after mastectomy, based on a descriptive epidemiological research. DATASUS data on the number of approved admissions and the total amount spent on treatment in patients undergoing simple mastectomy in oncology and radical mastectomy with axillary lymphadenectomy in oncology from June 2015 to June 2020 and patients undergoing plastic surgery were analyzed reconstructive breast after mastectomy with prosthesis implantation from June 2015 to June 2020. Data obtained through DATASUS reveal a total of 6,693 hospitalizations for surgical treatment of breast cancer and 1,185 hospitalizations for reconstructive breast plastic surgery post mastectomy with prosthesis implantation, showing that there is a low demand for reconstructive breast surgery by the Unified Health System. Mastectomy is an invasive process, which results in the mutilation of the breast, compromising the patient's body self-image and self-esteem. In this context, reconstructive surgery reduces the stigmas and sequelae left by mastectomy.
\end{abstract}

Keywords: Mastectomy; Mammoplasty; Breast neoplasms; Quality of life.

\section{Introdução}

Cânceré um conjunto de doenças caracterizadas pelo crescimento desalinhado de células, podendo invadir tecidos e órgãos. Nesse contexto, a divisão descontrolada de células da mamapode acarretar em características anormais que, associada a uma ou mais mutações do material genético da célula, dão origem ao câncer de mama ${ }^{1}$.

O câncer de mama é considerado um importante problema de saúde pública, sendo a neoplasia maligna mais incidente em mulheres na maior parte do mundo. No Brasil, estima-se que a incidência de casos novos no ano de 2019 tenha sido de 59.700, representando $29,5 \%$ dos cânceres em mulheres ${ }^{2}$. Nesse cenário, é essencial a detecção precoce e o adequado planejamento de estratégias de controle da doença, diminuindo a morbidade e a mortalidade associada ao câncer.

Há uma grande variedade de tipos histológicos e moleculares de carcinomas de mama in situ e invasor,

Universidade de Vassouras / Vassouras / Rio de Janeiro / Brasil. ORCID: https://orcid.org/0000-0002-0547-4614

${ }^{3}$ Universidade de Vassouras / Vassouras / Rio de Janeiro / Brasil. ORCID: https://orcid.org/0000-0003-2461-5854

${ }^{4}$ Universidade de Vassouras / Vassouras / Rio de Janeiro / Brasil. ORCID: https://orcid.org/0000-0001-8272-2071

${ }^{5}$ Universidade de Vassouras / Vassouras / Rio de Janeiro / Brasil. ORCID:https://orcid.org/0000-0002-4228-4641 
devendo ser sempre monitorados para avaliar sua disseminação e extensão e assim determinar o tratamento adequado $^{3}$. O principal objetivo do tratamento é erradicar o câncer, caso não seja possível, os objetivos passam a ser paliativos, como a melhora dos sintomas e a preservação da qualidade de vida. $\mathrm{O}$ tratamento do câncer de mama é dividido principalmente em cirúrgico, podendo ser conservador ou radical, radioterápico, quimioterápico e a terapia biológica ${ }^{4}$.

O diagnóstico do câncer de mama provoca um grande sofrimento para a mulher visto que ela passará a conviver constantemente com o medo, incertezas e ameaça com relação ao futuro, interferindo no seu estado emocional e acarretando em sofrimento e isolamento da paciente 5 . Esse quadro tende a se agravar com a mastectomia já que a mama é tida como um símbolo ligado a feminilidade e fortemente relacionado ao prazer, sensualidade, sexualidade e a maternidade ${ }^{6}$. A reconstrução da mama nessa perspectiva auxilia na recuperação da autoimagem e na superação da doença ${ }^{7}$.

O trabalho disserta sobre o acesso das mulheres mastectomizadas à cirurgia reconstrutora da mama pelo SUS visto sua grande contribuição para o bem-estar psicológico das pacientes.

O objetivo da pesquisa é comparar o número de internaçõeshospitalares entre a mastectomia oncológica e a plástica mamária reconstrutiva pós-mastectomia, enfatizando dessa forma a baixa procura para a cirurgia mamária reconstrutiva pelo Sistema Único de Saúde.

\section{Material e Métodos}

O presente trabalho trata de uma pesquisa do tipo epidemiológica descritiva. Os dados foram coletados no período de junho de 2015 a junho de 2020 . A população estudada esteve constituída pelas pacientes internadas pelo SUS, no estado do Rio de Janeiro, submetidas a cirurgia de mastectomia simples em oncologia e mastectomia radical com linfadenectomia axilar em oncologia no período de junho de 2015 a junho de 2020 e contabilizadas no formulário eletrônico do DATASUS.

O DATASUS é um Órgão da Secretaria de Gestão Estratégica e Participativa do Ministério da Saúde que coleta, processa e dissemina informações sobre saúde, proporcionando dados relacionados ao número de internações no sistema público hospitalar, bem como o tempo de internação, número de indivíduos tratados, forma de tratamento, taxa de mortalidade e montante gasto com tratamento. Neste trabalho, foram utilizados o número de internações aprovadas e o valor total gasto com tratamento em pacientes submetidas a mastectomia simples em oncologia, mastectomia radical com linfadenectomia axilar em oncologia e mastectomia radical com linfadenectomia no período de junho de 2015 a junho de 2020 e pacientes submetidos a plástica mamária reconstrutiva pós-mastectomia com implante de prótese no período de junho de 2015 a junho de 2020.

Associado a isso, foi efetuado uma busca sistemática de artigos, sem restrição de tempo, dados dos sistemas de informação do Instituto Nacional de Câncer (INCA) e o livro de Hematologia e oncologia do autor Harrison, $2^{\circ}$ edição, e assim aprofundar sobre o câncer de mama, seu tratamento, suas implicações físicas e emocionais e a utilização da mamoplastia reconstrutiva pós-mastectomia.

\section{Resultados}

A partir dos dados colhidos do DATASUS, foram analisadas as internações por mastectomia radical com linfadenectomia axilar em oncologia, mastectomia simples em oncologia, mastectomia radical com linfadenectomia, plástica mamária reconstrutiva pósmastectomia com implante de prótese; além do valor total gasto com cada procedimento.

É sempre importante ressaltar a importância do rastreamento e do diagnóstico precoce do câncer de mama. Segundo a Organização Mundial da Saúde, cerca de um terçodos cânceres podem ser curados caso sejam detectados e tratados precocemente ${ }^{8}$. Em relação a detecção precoce, é essencial a conscientização da população e capacitação dos profissionais de saúde sobre os sinais e sintomas do câncer de mama e, assim, promover o diagnóstico o mais breve possível dos pacientes sintomáticos.

Nesse contexto, o autoexame das mamas pode ser uma ferramenta importante nessa detecção ${ }^{9}$, porém o Ministério da Saúde não recomenda como técnica a ser ensinada uma vez que estudos revelaram não afetar os índices de mortalidade. Contudo, é estimulado que a mulher possua uma postura atenta no reconhecimento de alterações suspeitas em seu corpo, como mudanças na pele ou formato da mama, saída de secreções do mamilo, nódulos no pescoço ou na axila, dor na região e áreas hiperemiadas, sendo considerada estratégia importante para o diagnóstico precoce do câncer de mama ${ }^{1}$.

Orastreamento é feito em pacientes assintomáticas na faixa etária de 50 a 69 anos, a cada dois anos, através da mamografia, que permite identificar melhor as lesões mamárias em mulheres mais velhas através da classificação de BI-RADS ${ }^{10}$ (Quadro 1).

Uma vez realizado o diagnóstico, o meio mais efetivo de tratar o câncer é através da cirurgia, porém, mesmo quando a doença não é curável, a retirada do tumor traz inúmeros benefícios como o melhor controle da doença, a diminuição do volume da massa e a melhor resposta a terapia subsequente ${ }^{4}$.

A intervenção cirúrgica, conhecida como mastectomia, pode ser limitada a retirada do tumor e, 


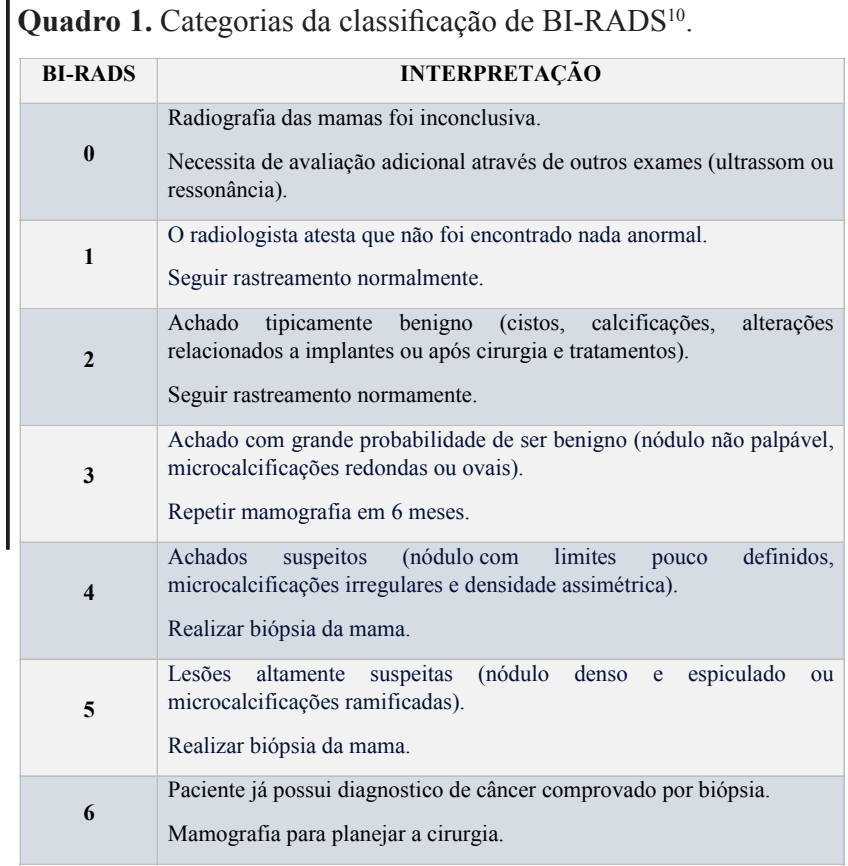

Legenda: BI-RADS: Breast Imaging Reporting and Data System

caso tenha atingido tecidos mais profundos, envolve a retirada da mama, dos linfonodos axilares e do tecido muscular. A mastectomia radical modificada, na qual remove toda a mama e os linfonodos axilares, é a mais corriqueira já que normalmente a doença é diagnosticada em estágios mais avançados. Tratamentos adjuvantes podem ser necessários, como a quimioterapia, a radioterapia e a hormonioterapia ${ }^{11}$.

A cirurgia reparadora da mama representa um grande avanço para as pacientes que passam pelo câncer de mama. Uma das vantagens para a mulher é o alívio psicológico e estético de ter sua mama reconstruída e, assim, recuperar a integridade corporal e autoestima ${ }^{7}$.

No período de junho de 2015 a junho de 2020, no estado do Rio de Janeiro, foramcontabilizadas4.349 internações para realização de mastectomia radical com linfadenectomia axilar em oncologia, 1.493 internações para realização de mastectomia simples em oncologia e 851 internações para realização de mastectomia radical com linfadenectomia, totalizando 6.693 internações para realização do tratamento cirúrgico do câncer de mama. Comparativamente, foram efetivadas 1.185 internações para a realização de plástica mamária reconstrutiva pósmastectomia com implante de prótese (Figuras 1 e 2).

$\mathrm{O}$ valor total gasto com as cirurgias de mastectomia foi de $\mathrm{R} \$ 16.562 .173,56$ enquanto o valor total gasto com as plásticas mamárias foi de R $\$$ $808.332,91$.

\section{Discussão}

Ao correlacionar o conteúdo abordado na literatura aos dados colhidos,notou-se que os tratamentos

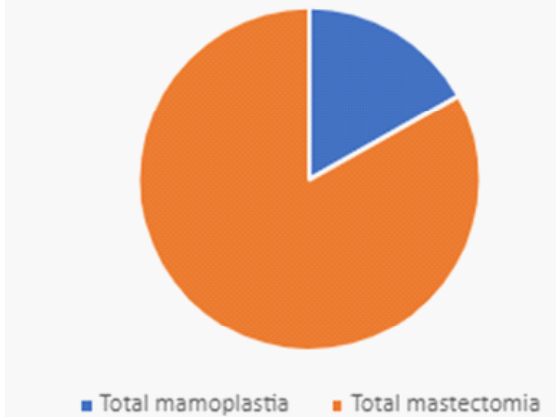

Figura 1. Comparação de mastectomias em oncologia x cirurgia plástica mamária reconstrutiva pósmastectomia com implante de prótese.

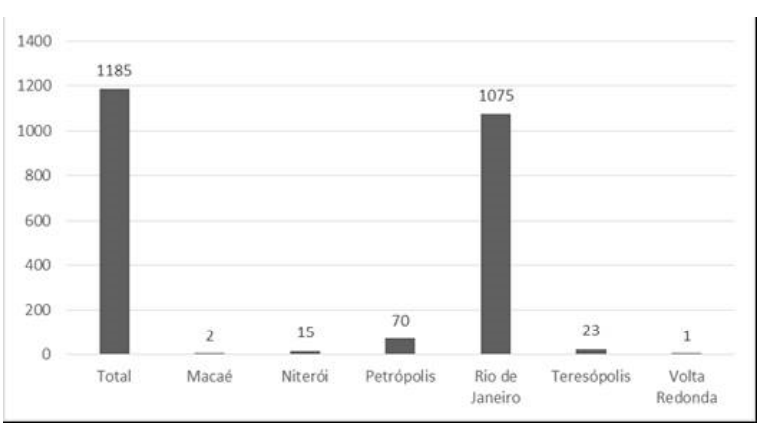

Figura 2. Internações para plástica mamária reconstrutiva pós-mastectomia com implante de prótese no Estado do Rio de Janeiro de junho de 2015 a junho de 2020.

são subdivididos em local, abrangendo cirurgia e radioterapia, e sistêmico, incluindo quimioterapia, hormonioterapia e terapia biológica. As diretrizes revelam que nos tumores in situ, o tratamento local foi utilizado em $62,6 \%$ e, o tratamento local e sistêmico combinado foi utilizado em $32,9 \%$. Nas pacientes com estadiamento nos grupos I, II e III, o tratamento combinado chegou a $70 \%$ e nos pacientes com estádio IV , 47,2\% foram submetidos ao tratamento sistêmico isolado ${ }^{3}$. Nos dados analisados, notou-seque a cirurgia, além de ser o tratamento mais efetivo no câncer de mama, é fortemente recomendado pelo Instituto Nacional de Câncer (INCA) na maioria dos casos.

É importante ressaltar que as mamas desempenham importante papel fisiológico no desenvolvimento feminino, além de ser um símbolo da feminilidade. A mastectomia se trata de um processo invasivo, que acarreta na mutilação da mama, comprometendo a autoimagem corporal e causando uma sensação de inadequação à paciente, podendo gerar isolamento, depressão e dificuldades de retornar às atividades prévias à cirurgia ${ }^{12}$. As principais aflições envolvem à perda da sexualidade, a alteração da imagem corporal, o afastamento do parceiro e a morte ${ }^{13,14}$. Nesse contexto, a cirurgia reconstrutiva reduz os estigmas e sequelas deixadas pela mastectomia, auxiliando no 
bem-estar psicológico e na autoestima, suprindo a falta da mama e facilitando o uso de vestuário ${ }^{7}$.

O tipo de procedimento cirúrgico também contribui fortemente para a qualidade de vida do paciente oncológico e deve ser avaliado. A mastectomia radical consiste na remoção total da mama, dos músculos peitorais, da pele e dos gânglios linfáticos axilares. Como uma alternativa menos invasiva, a mastectomia radical modificada foi introduzida e difere por preservar o músculo do grande peitoral. A introdução das técnicas cirúrgicas de conservação da mama, como a mastectomia simples, mastectomia poupadora da pele e mastectomia poupadora do mamilo, seguida de radioterapia tem se mostrado uma boa opção para o tratamento de câncer de mama nos estágios iniciais ${ }^{15}$.

Os dados obtidos através do DATASUS revelam que existe uma baixa procura para a cirurgia mamária reconstrutiva pelo Sistema Único de Saúde (SUS) e/ou acesso aos serviços no estado em questão. Esses dados tem inúmeros motivos, como a falta de orientação pela equipe médica sobre essa possibilidade, o medo de passar por uma cirurgia que pode acarretar em sofrimento e rejeição da prótese e a possibilidade de complicações após a técnica ${ }^{13}$.

É fundamental para o restabelecimento da paciente uma participação efetiva da rede sociofamiliar para a compreensão do processo que está vivendo, bem como para ajudar a lidar com os conflitos e repercussões causados pela doença ${ }^{14}$. Associado a isso, é necessária uma equipe multidisciplinar atenta e capacitada formada por médicos, enfermeiros, fisioterapeutas, psicólogos e assistência social que busquem resgatar a paciente e propor o melhor tratamento.

\section{Considerações finais}

O câncer de mama é a neoplasia maligna mais incidente em mulheres na maior parte do mundo sendo o tratamento cirúrgico o meio mais efetivo de tratar o câncer. A descoberta de uma neoplasia mamária com a realização de uma mastectomia promove uma intensa mudança na vida da mulher, relacionadas principalmente com a perda da autoestima, o isolamento social e o medo da morte.

A reconstrução da mama e as técnicas cirúrgicas mais conservadoras podem promover uma melhora na qualidade de vida, auxiliando na preservação da autoestima, aceitação da autoimagem e em uma reabilitação menos traumática. Apesar disso, apenas cerca de $17,7 \%$ das pacientes mastectomizadas são encaminhadas para a realização da plástica mamária reconstrutiva pós-mastectomia. Acredita-se que isso decorre principalmente da falta de orientação adequada sobre o assunto e o receio da paciente de passar por uma nova cirurgia.
Os artigos demonstram que além da satisfação estética, a mamoplastia acarreta na diminuição do índice de morbidade psicológica sendo, portanto, um importante recurso na reabilitação das pacientes que são submetidas a uma mastectomia.

\section{Referências}

1. O que é câncer? [homepage Internet]. Instituto Nacional de Câncer; [acesso em 19 ago 2020]; Disponível em: https://www.inca.gov.br/o-que-ecancer

2. Instituto Nacional de Câncer José Alencar Gomes da Silva (INCA) [homepage na internet]. A situação do câncer de mama no Brasil: Síntese de dados dos sistemas de informação [acesso em 24 de ago 2020]. Disponível em: https://www.inca.gov.br/sites/ufu.sti.inca.local/files/media/document/a situacao ca mama brasil 2019.pdf

3. Santichi EC, Benute GRG, Juhas TR, Peraro EC, Lucia MCSd. Rastreio de sintomas de ansiedade e depressão em mulheres em diferentes etapas do tratamento para o câncer de mama. Psicologia Hospitalar. 2012;4267

4. Longo DL. Hematologia e Oncologia - Harrison. $2^{\mathrm{a}}$ ed. Universidade Federal de São Paulo (Unifesp): AMGH editora Ltda; 2015

5. Silva LC. Câncer de mama e sofrimento psicológico: aspectos relacionados ao feminino. Psicologia em Estudo 2008;231-237.

6. Martins MM, Peres RS. Fatores terapêuticos em grupo de apoio a mulheres com câncer de mama. Psic., Saúde \& Doenças. 2014;15(2): 396408

7. Inocenti A, Santos MA, Loyola EA, Magalhães PA, Panobianco MS Repercussão dos efeitos da cirurgia reconstrutora na vida de mulheres com neoplasia da mama. Texto Contexto Enferm. 2016; 25(2)1-9.

8. Silva RC, Hortale VA. Rastreamento do Câncer de Mama no Brasil: Quem, Como e Porquê? Revista Brasileira de Cancerologia. 2012; 58(1): $67-71$.

9. Müller MC, Frasson A, Kieling C, Hoffmann FS, Fleck P, Zogbi H, et al. A prática do auto-exame das mamas em mulheres de uma comunidade universitária. Psico-USF. 2005;185-190.

10. Pereira MB, Oliveira J, Ribeiro DP, Castro B, Yaphe J, Souza JC, et al. Grupo etário e periodicidade recomendados para a mamografia de rastreio: uma revisão sistemática. Ciência \& Saúde Coletiva. 2014;1135-1140.

11. Barros AC, Barbosa EM, Gebrim LH, Anelli A, Figueira A, Del Giglio A, et al. Diagnóstico e Tratamento do Câncer de Mama. 2001. 1-15.

12. Ferreira DC, Lima DM. As Intervenções na Psicologia para a Preparação e Acompanhamento de Pacientes Submetidos à Mastectomia. Psicologado. 2018. Disponível em: https://psicologado.com.br/atuacao/ psicologia-hospitalar/as-intervencoes-na-psicologia-para-a-preparacao-eacompanhamento-de-pacientes-submetidos-a-mastectomia

13. Braganholo LP. A não-realização de cirurgia reconstrutiva de mama: fatores associados, qualidade de vida e autoestima. Ribeirão Preto. Dissertação [Mestrado em enfermagem em saúde pública]. Escola de enfermagem de Ribeirão Preto, Universidade de São Paulo; 2007

14. Gonçalves SR, Arrais FM, Fernandes AF. As implicações da mastectomia no cotidiano de um grupo de mulheres. Rev Rene [revista em Internet].2007 maio [acesso 19 de agosto de 2020];9-17. Disponível em: http://repositorio.ufc.br/handle/riufc/13561

15. Moreira H, Canavarro MC. Tipo de cirurgia, adaptação psicossocial e imagem corporal no cancro da mama. Psicologia, Saúde \& Doença. 2012. 13(2):169-190. 